\title{
Zoonotic impact and epidemiological changes of leishmaniasis in Ethiopia
}

\author{
Dawit Gebremichael* \\ Veterinary Public Health, Aksum University Shire Campus, College of Agriculture, Department of Animal Science, \\ Shire, Ethiopia
}

\begin{abstract}
Leishmaniasis is one of the growing public health challenges in Ethiopia and estimated over 7,000 and 50,000 new cases of visceral leishmaniasis (VL) and cutaneous leishmaniasis (CL) per year, respectively. The aim of the review is to address zoonotic impact and epidemiological changes of leishmaniasis in Ethiopia. VL is caused by L. donovani and it is endemic in many parts of the country with one third of the country's landmass is highly suitable for VL. CL is principally caused by L. aethiopica. CL is endemic and widespread in the highland of Ethiopia. Northern lowland foci are Humera and Metema plains in the Tigray and Amhara regional states constitute the main VL endemic areas in the country, contributing over $60 \%$ of the total burden. The southern foci are the south-western savannah, and the south-eastern semi-arid lowlands which account for approximately $20 \%$ of the total VL burden in Ethiopia. Leishmaniasis is a serious zoonotic disease in Ethiopia with more reservoir hosts maintaining the disease. Dogs and hyraxes are the main reservoir hosts for visceral and cutaneous leishmaniasis in the country, respectively. Epidemiological changes of leishmaniasis may relate to environmental changes and expansion of mega projects such as irrigations and sugar cane factories, knowledge and socio-economic factors, development of new settlements, migration of peoples and HIV/AIDS co-infection. Expansions of mega projects such as sugar factories and irrigations are suitable for reproduction of stray dogs, rodents, wild canids and vectors. VL is one of the major challenges to prevent and control in the endemic areas of the country. Therefore, new research should be imperative, especially in the mega projects to design strategic control and prevention methods.
\end{abstract}

Keywords: Environmental change, Epidemiological change, Leishmaniasis, Reservoir hosts, Zoonotic.

\section{Introduction}

Leishmaniasis is the most neglected vector borne diseases and can be caused by several species of obligate intracellular protozoan of the genus Leishmania. This disease is endemic in several tropical, subtropical and Mediterranean basins (Assimina et al., 2008; CFSPH, 2009; Hailu et al., 2016; Oryan and Akbari, 2016). Recently, leishmaniasis has been emerged or reemerged in many geographical areas, making global health and economic concerns that could be involved in humans, domestic animals and wildlife (Ready, 2008; Coura-Vital et al., 2013; Herrador et al., 2015; Oryan and Akbari, 2016). Leishmaniasis is transmitted by the bite of phlebotomies female sand flies of the genera Phlebotomus and Lutzomyia, in the old and new worlds, respectively (Reithinger et al., 2010; Dawit et al., 2013; Savoia, 2015).

Leishmaniasis is endemic in 98 countries with greater than 350 million people at risk and an estimated 700 000-1.2 million new cases of which, 600000 to 1 million new cases of CL, 50000 to 90000 new cases of VL and about 60,000 deaths from both CL and VL each year (Collin et al., 2006; Oryan et al., 20014; WHO, 2015; Oryan and Akbari, 2016; Spear, 2017). Of the entire current VL incidence reported, about $90 \%$ cases are from seven countries (Brazil, Ethiopia, India, Kenya, Somalia, South Sudan and Sudan). Similarly, the majority of CL cases are endemic in only ten countries (Afghanistan, Algeria, Brazil, Colombia, Ethiopia, Iran, Pakistan, Peru, Saudi Arabia and Syria) (Desjeux, 2004; Murray et al., 2005; Lemma et al., 2014; WHO, 2014; Hailu et al., 2016, Lemma, 2018). Eastern Africa is the second largest VL foci after the Indian subcontinent, contributes to the global burden with 30,000-40,000 new cases per year (Leta et al., 2014).

In Ethiopia, VL mainly occurs in the arid and semi-arid lowland areas. However, recent reports indicated that the spreading of the disease in areas where it was previously non-endemic (Leta et al., 2014). Environmental modifications, socio-economic status, expansion of agricultural mega projects and new reservoir hosts are the most important risk factors of the disease (Assimina et al., 2008; Gadisa et al., 2015). A study was conducted in Ethiopia to identify environmental parameters influencing the geographical distribution of VL. According to Leta et al. (2014), annual average temperature showed a high leverage on VL occurrence. Recent spread beyond the Metema and Humera plains to villages in Tahtay Adiabo, Sheraro,

*Corresponding Author: Dawit Gebremichael. Veterinary Public Health, Aksum University Shire Campus, College of 
Welkait and Armacheho due to environmental changes. Therefore, the aim of the review is to address epidemiological changes, zoonotic impact and public health threats of leishmaniasis. The available evidence from various studies including research articles, review articles, textbooks and standard guidelines were retrieved from PubMed, African Journal Online, Scopus databases and Google scholar.

\section{Status of leishmaniasis in Ethiopia}

The annual burden of VL in Ethiopia is estimated to be between 4,500 and 7,400 cases (Lemma, 2018), and the annual incidence of the CL range from 20,000 to 50,000 cases, but this is probably an under-estimate (Kassahun et al., 2015a). The population at risk is more than 3.2 million and 28 million people inhabit in regions with risk of VL and CL, respectively (Alvar et al., 2012; Leta et al., 2014; Seid et al., 2014; Lemma, 2018). Incidence rates of VL and CL cases per 10000 population in endemic areas are 6.28 and 1.05, respectively (WHO, 2017). The incidence rates of VL and CL every year in Ethiopia are presented in Fig. 1 and 2.

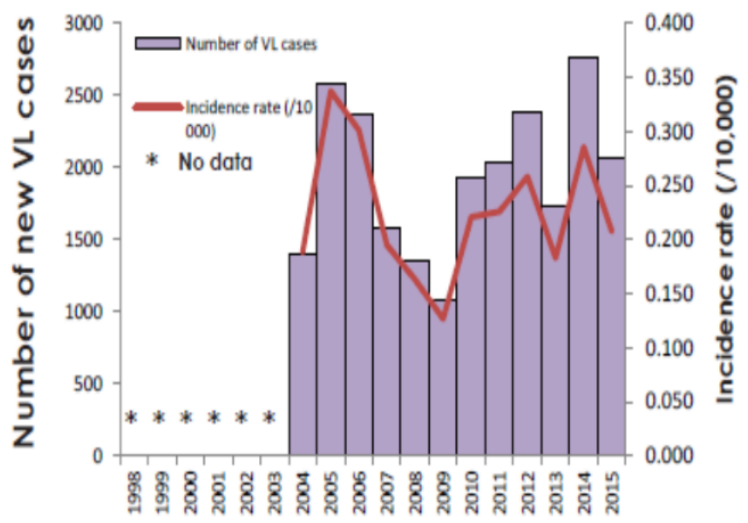

Fig. 1. Incidence rates per 10,000 and number of new cases of VL from 1998 to 2015 in Ethiopia. Source: (WHO, 2017).

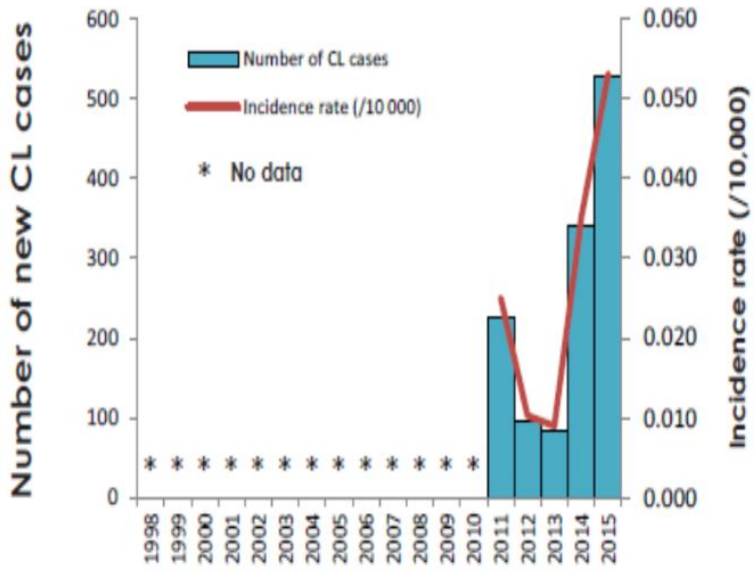

Fig. 2. Incidence rates per 10,000 and number of new cases of CL from 1998 to 2015 in Ethiopia. Source: (WHO, 2017).
Ethiopia has the second largest number of annual VL case of Africa, next to Sudan. VL is caused by $L$. donovani and it is endemic in many parts of the country with one third of the country's landmass is highly suitable for VL (Gadisa et al., 2015). CL is principally caused by $L$. aethiopica and it is endemic and widespread in the highland areas of the country. Though rarely, L. tropica and L. major have also been implicated in the lowland regions.

The main foci are found in the lowland areas of north, northwestern, and southwestern Ethiopia, with some sporadic cases in the central-east part of the country (Kassahun et al., 2015a). Northern lowland foci are Metema and Humera plains in the Tigray and Amhara regional states, bordering Sudan and Eritrea, constitute the main VL endemic areas, contributing over $60 \%$ of the total burden in the country (Dawit et al., 2013; Leta et al., 2014; Lemma et al., 2015). The other main focus in the southwest is in the lower course of the Rift Valley, most notably, the Segen (Aba Roba focus) and Weyto Valleys in the drainage basin of the Chew Bahir Lake, near Konso Woreda. The Aba Roba focus has a particularly high VL endemicity and high population immunity, with $36.4 \%$ testing positive with the leishmanin skin test (Leta et al., 2014; Gadisa et al., 2015). Further eastward, in the Oromia regional state, sporadic VL cases have been reported from Lake Abaya, Moyale area, Dawa and Genale River Valleys. Down in the south-eastern semi-arid lowlands, there is a recent report of VL transmission in the Afder and Liben areas in the Somali regional state ((Leta et al., 2014; Gadisa et al., 2015).

Cutaneous leishmaniasis has been occurred in the highlands of Ethiopia. Cutaneous form has been extensively studied in the western highlands and lake areas of the Rift Valley. The main areas of transmission include the Ochollo focus in the Rift Valley escarpment above Lake Abaya, the Kutaber area in the eastern Ethiopian plateau near Dessie, the Aleku area of Wollega zone, the south-west highlands of Bale and Sidamo, the Sebeta area near Addis Ababa and the eastern Tigray highland of Adi-grat and Saesie Tsaedaemba district (Dawit et al., 2013, Bsrat et al., 2015; Kassahun et al., 2015a).

\section{Zoonotic impact}

\section{Reservoir hosts and sources of infection}

Leishmaniasis is a serious zoonotic disease with one or more reservoir hosts maintaining the disease. Mammalian reservoir hosts are responsible for maintaining in a population of an infectious agent in Ethiopia for a long period of time. It could be due to zoonotic nature of leishmaniasis in Ethiopia, where drug case management could not decrease incidences of leishmaniasis as humans are incidental (dead end) hosts (Lemma, 2018). Leishmania species differ in the degree to which they are associated with different host 
species and reservoirs. However, their role in the transmission cycle as a reservoir host and source of infection in human is significantly different with in the country (Lemma, 2008; Argaw et al., 2013; Alemayehu and Alemayehu, 2017).

\section{Dogs}

Dogs are the most important species among domestic animals in the epidemiology of this disease; hence, stray and domestic dogs are potential reservoir hosts for L. donovani in Ethiopia (Dereure et al., 2000; CDC, 2013; Dawit et al., 2013; Leta et al., 2014; Al-Salem et al., 2016). Increasing the incidence rate of VL in endemic areas has been associated with residents who slept with dogs (Argaw et al., 2013). Recently, a natural infection of dogs with $L$. donovani complex was reported in Ethiopia (Kassahun et al., 2015b). Dogs owned by people with a history of VL have a higher risk of infection when compared to cattle in Humera regions (Gadisa et al., 2015). Perhaps cattle play no role as reservoirs of $L$. donovani, but they may attract the vector to human houses and thereby increase the risk of human exposure to infected sand flies.

\section{Hyraxes}

In Ethiopian highlands, where the CL is endemic and the ecological and behavioral requirements of hyraxes make them perfect reservoir hosts of $L$. aethiopica (Lemma, 2008; Lemma, 2018). Rock hyraxes were found infected with L. aethiopica, suggesting a zoonotic cycle of the parasite (Ashford et al., 1973; Kassahun et al., 2015b). L. aethiopica is naturally harbored by two species of rock hyraxes (Procavia capensis and Heterohyrax brucei); however, it has been believed that diverse groups of hyraxes could be involved in the epidemiology of CL (Lemma et al., 2009). In the highland areas, intense outbreaks of CL are usually associated with the existence of hyraxes. Thus, there is no doubt about zoonotic transmission of CL in Ethiopian highlands (Lemma, 2018). According to Bsrat et al. (2015), higher prevalence was reported from those whose habitats were near to hyrax shelter and the disease seems to be of zoonotic nature. Rock hyraxes are also epidemiological proven reservoirs of several Leishmania species that are found repeatedly naturally infected (Kassahun et al., 2015b).

\section{Other reservoir hosts}

The L. tropica and L. major positive bats were found in areas where potential vectors occur in Ethiopia (Kassahun et al., 2015b). Bats could have adequate features to be naturally infected by Leishmania parasites and could subsequently play a role in its epidemiological cycle. The natural Leishmania infection of bats is recorded in both endemic and nonendemic areas of CL in Ethiopia (Kassahun et al., $2015 b$ ). In addition, wild canids and rodents are also the main reservoir hosts in the country (Kassahun et al., 2015a), from the rodent, Arvicanthis niloticus is reservoir host for L. major. In VL endemic lowland areas, the suspected rodent reservoir hosts which were found infected with Leishmania parasites exist abundantly in pre-domestic and extra-domestic habitats. Rodents, most probably, have maintained the zoonotic L. donovani parasites cycle in all VL endemic foci (Lemma, 2018). These and previous evidences from the endemic foci of Ethiopia showed that domestic mammals (sheep, goats and donkey) in endemic area are possibly having role in epidemiology of VL (Kenubih et al., 2015).

\section{Epidemiological changes of leishmaniasis}

Environmental changes and expansion of sugar factories

Environmental change will often modify the transmission patterns of vector-borne diseases, through its effects at different ecological scales. Improving vegetation types and climate condition were favored to increase vector densities or host-vector contacts and reintroductions of pathogens, or breakdown of vector control measures (Yared et al., 2014). The existing major agricultural schemes, ongoing and planned mega-projects such as sugar industries and sugar cane farming, dams for electricity/irrigation purposes and expansion of irrigation and/or rain fed agriculture both by small scale farmers and large scale investors all exist within the lower Kola to Weina Dega agro-ecological zones. The infection rate in the zoonotic cutaneous and visceral leishmaniasis is often highest among people living at the edge of environmental modified (WHO, 2010). Thus, the re-emergence of VL in Ethiopia will have public health threat and social implications (Gadisa et al., 2015). Expansions of mega projects such as sugar factories and irrigations are suitable for reproduction of stray dogs, rodents, wild canids and vectors. At the same time, movements of people are also increased to the area. Currently, there are ten ongoing new sugar cane irrigations/factories in Ethiopia; however, there is no clear data or any research concerning the mega projects and distribution of leishmaniasis in Ethiopia.

In the western zone of Tigray, new agricultural irrigation is existed for sugar cane industries and farming purposes near to VL endemic areas. This modification is important to increase the population of reservoir hosts and vectors. Changes in land coverage such as deforestation and continually replace by irrigation and farmlands has an increasing risk for leishmaniasis. As the population of the reservoir hosts such as dogs, wild canids and rodent increases, the prevalence and incidence rate of VL has increased and the sandfly vectors have become peri-domestic (WHO, 2010; Leta et al., 2014).

Welkait sugar factory is one of the ongoing mega projects which is located in northwestern Tigray which is bordered by Sheraro and Tahtay Adiabo. This area is 
adjacent to VL endemic areas of Humera, western Tigray (Dawit et al., 2013; Leta et al., 2014). Recent spread beyond the Humera plains to Welkait, Sheraro and Tahtay Adiabo, northwestern Tigray. Especially in villages of Sheraro VL claimed the lives of hundreds and remains circulating since its first investigation $(\mathrm{FMoH}, 2013)$. The new focus in the semi-arid lowlands of Tahtay Adiabo and Sheraro district of northwestern Tigray became the most important focus in the epidemiology of VL (Gebresilassie et al., 2015). This might be due to the existing new agricultural irrigation for Welkait sugar factory. According to the hospital reports of the Welkait district health office, there was a visceral leishmaniasis (VL) outbreak in 2013 in a specific area called Korarit sub-district, making the disease a public health concern for the regional health authorities ever since (Berhe et al., 2018).

Kuraz is one of ongoing sugar factories and has three projects in some selected areas of Omo, Southern Ethiopia and has identified as sites of huge potential for sugar development. However, the southern foci are the south-western savannah, and the south-eastern semiarid lowlands which account for approximately $20 \%$ of the total VL burden in Ethiopia lay in Omo valley (Malaria Consortium, 2010; Leta et al., 2014; Gadisa et al., 2015). The construction of a bridge on a gorge that bisects a "Silti" town in Southern Ethiopia has created an ideal environment for sand flies and hyraxes to reproduce underneath. This resulted in a rapid population growth of sandflies and hyraxes and in the emergence of CL in the area (Negera et al., 2008). In a situation like this, where human-made environmental change results in increased risks for leishmaniasis. Temperature, rainfall and humidity may changes due to global warming, influencing survival and population size of vectors and reservoir hosts. The unique human adaptation and settlement patterns of the communities in these areas and the very focal nature of sandfly habitats are believed to be the major factors for the local variations in the burden of VL in the valleys (Leta $e t$ al., 2014).

A Tendaho sugar factory is also one of the newest projects located in Awash Valley, Afar region. In the lowlands of Awash Valley, VL occurs with high population of immunity. Leishmanin skin test rates remain high in this area. About $59.5 \%$ of VL was reported among Afar pastoralists (Fuller et al., 1976; Ali and Ashford, 1994). In wide areas of the northeastern Rift Valley of Ethiopia, sporadic VL cases have also been recorded, mainly in the Awash Valley and Afar regional states. But nowadays, VL was reported in Afder district. This may be due to establishment of new agricultural projects, irrigation, storage of waste products and increase sand flies population (Leta et al., 2014).
Wonji shewa and Metehara are planted in the upper Awash Valley.

In Metehara town, overall incidence of visceral leishmaniasis was $22.6 \%$ in the last five years (20082012); and the trend of VL has been increased in this district (Shiferaw et al., 2016). Available data in the Metema Hospital from 2008 to 2012 showed that the incidence rate was higher in the age group of 15-29 (Hailu et al., 2009; Malaria Consortium, 2010). This might be associated with their daily activities such as extensive farming that young daily laborers move to Metema irrigation from different areas (Getahun and Belete, 2007; Zackay et al., 2013; Wondimeneh et al., 2014; Shiferaw et al., 2016). The epidemiological distribution of visceral leishmaniasis is presented in Fig. 3.

\section{Knowledge and socio-economic factors}

Leishmaniasis, especially the visceral form, tends to affect the poorest people and marginalized societies. $\mathrm{VL}$ is concentrated in poverty-stricken districts of the country (Leta et al., 2014).

Poor protein, energy, iron, vitamin $\mathrm{A}$, and zinc nutritional status was recorded as a high risk of VL cases in Ethiopia (Leta et al., 2014). Poor housing and sanitary conditions such as lack of waste management, open sewerage and poor health facilities are an indication of poverty. Thus poverty increases the risk of VL through increasing the breeding of sand flies and their access to people (Yared et al., 2014; Hailu et al., 2016).

Moreover, low educational level and socioeconomic concerns also increases risks of both VL and CL in the country (Dawit et al., 2013). According to Bsrat et al. (2015), the knowledge on the causative agent, source and mode of transmission of CL in Tigray region was limited. Knowledge, attitude and practice towards VL in Humera were very poor in the rural communities of Humera. People with poor knowledge may increase the exposures of VL and CL than people having awareness.

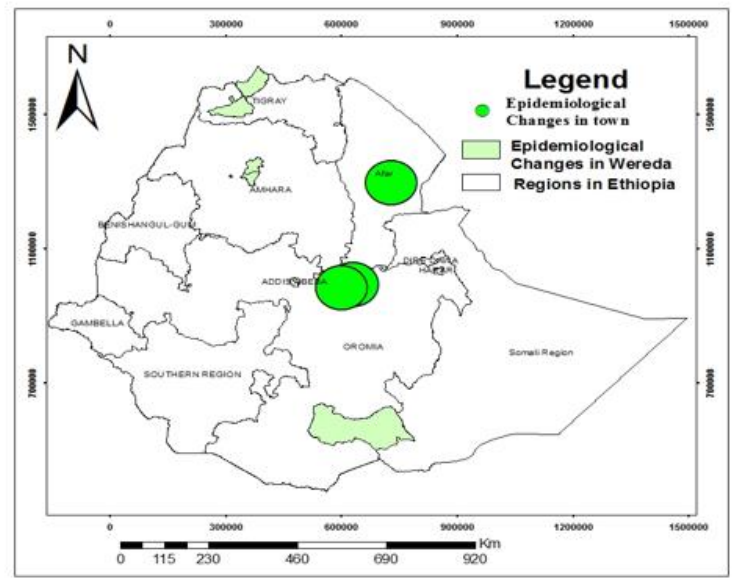

Fig. 3. Epidemiological changes of leishmaniasis. 


\section{Population activities}

Social and political forces, especially military activities and agricultural development projects are major drivers of VL endemicity and new epidemics in Ethiopia (Leta et al., 2014; Lemma et al., 2015; Al-Salem et al., 2016). The population movements probably lead to alterations in the number, range and density of the vectors and reservoirs consequently.

The changing epidemiology of VL in northern Ethiopia could most probably be linked to this factor (Leta et al., 2014). The agricultural activities such as weeding and harvest of sesame and sorghum in Humera, western lowlands Tigray attract around 200,000 seasonal labor migrants annually, mainly from the surrounding Amhara and Tigray highland areas. In this endemic region, VL particularly affects migrant workers (Dawit et al., 2013; Lemma et al., 2015).

\section{HIV co-infection}

Co-infection with the human immunodeficiency virus (HIV) is among the factors believed to contribute to reemergence of VL in Ethiopia (Chappuis et al., 2007). Probably the biggest challenge in the control of VL in Ethiopia is its association with HIV/AIDS, coupled with the seasonal migration of laborers to and from endemic areas (Dawit et al., 2013).

In northwest Ethiopia, up to $23 \%$ of VL cases are HIV co-infected in 2008, far higher than anywhere else in the world (Tsegaw et al., 2013). Hospital based studies in the northern western Tigray, mainly serving migrant laborers, documented an alarming increase of HIV/VL co-infection is from $29 \%$ to $46.4 \%$ in 2006 to 2010 (Hurissa et al., 2010; Gadisa et al., 2015). Around 60\% of VL incidence was reported in the highest HIV prevalence among all leishmaniasis patients from northwest Ethiopia (Lemma, 2018).

\section{Vectors}

$P$. martini, $P$. celiae and $P$. orientalis have been confirmed the main vectors of VL in Ethiopia (GebreMichael et al., 2010). P. longipes and P. pedifer commonly identified vectors of $L$. aethiopica from different parts of the county (Ashford et al., 1973). P. orientalis is the most probable vector in Humera and Metema endemic areas of Ethiopia and nearby Sudanese foci (Moncaz et al., 2013). In southern Ethiopia, P. martini and P. celiae are believed to transmit the disease (Shabtai et al., 2014). However, in other places, like Tahtay Adiabo foci, $P$. orientalis is associated with different microhabitat (Gebresilassie $e t$ al., 2015).

Distribution of $P$. orientalis was associated with maximum daily temperature, soil type (black cotton clay) and Acacia-Balanites woodland of the savanna. $P$. orientalis has been observed in very lowland, the presence of Acacia-Balanites vegetation and cracks in black cotton clay soil (Gadisa et al., 2015). P. orientalis shifts from black cracking soil in agricultural fields to hollows in tree trunks in dense mixed forest near farms (Lemma et al., 2014). Entomological studies at the highland VL focus of Libo Kemekem district showed that $P$. orientalis had potential to play a role for the establishment of infection mainly due to its high abundance and even in many sites being the only Phlebotomus species out of the catches (Gebre-Michael et al., 2007).

\section{Control and prevention}

Government of Ethiopia, particularly Tigray regional state, has developed its own control strategies so as to limit the rapid spread of the disease. A National leishmaniasis task force was established in 2007 with the aim of eliminating VL and Hospitals and health centers in endemic regions equipped to treat VL include: Kahsay Abera Humera Hospital, Aksum Hospital and Mekelle Hospital in Tigray regional state. The best control strategies for leishmaniasis rely on reservoir and vector control (Kishore et al., 2006; Chappuis et al., 2007), improved health education (Leta et al., 2014), and early identification and management are essential for both individual patients and for the community (Hide et al., 2007; WHO, 2010). However, vectors and reservoirs control have never been practiced in the control of leishmaniasis in Ethiopia, and no convincing study was conducted to determine the role of sandfly control in the transmission dynamics of VL.

\section{Control of reservoir hosts}

Control of reservoir hosts have been recommended as a component of control strategies for zoonotic visceral and cutaneous leishmaniasis (WHO, 2010). In the Old World, identification and control of animal reservoirs (small rodents) consist of deep plowing to destroy the burrows (breeding and resting sites) and plant (Chenopodiacae) sources of food for the rodents (Hide et al., 2007). Dogs are the main reservoir of $L$. donovani in the zoonotic VL in Ethiopia. The effective methods include culling of stray dogs and management of dog population to control VL. The elimination of stray and feral dogs is justified for many reasons connected with health, the environment and conservation (WHO, 2017). Controlling the population of stray dogs is also important to control of other zoonotic diseases such as rabies and echinococcosis. Wild rodents in Ethiopia could play an important epidemiological role in the transmission cycle of two Leishmania species, L. donovani and L. tropica (Kassahun et al., 2015a). The reservoir hosts of CL (hyraxes) live mostly in crevices of basalt rocks in the gorges and tree cavities in forests and trapping, diagnosis and culling or treating infected hyraxes is almost impossible. However, it might be possible to control CL due to L. aethiopica by shooting on hyraxes close to heavily infected villages or by encouraging specific predators such as the eagle, mongoose, genet 
and cat (Lemma, 2008). Control of hyraxes around villages may reduce incidence of leishmaniasis. Elimination of hyraxes within $1 \mathrm{~km}$ of settlements is thought to be effective in reducing transmission (Getachew et al., 2006; WHO, 2010).

Vector control

Vectors control is an effective strategy for minimizing the incidence of leishmaniasis. Sandfly population should be reduced through application of chemicals, environmental management and personal protection (Hailu et al., 2016). Environmental management and chemical control are reduced sandfly population. Physical modification of the sandfly habitat, relocation of human settlements away from known vector habitats, and the implementation of environmental sanitation programs are very important to eliminate actual or potential breeding sites to control both malaria and VL in the country (FMoH, 2013).

In areas where Leishmania transmission occurs outdoor and where leishmaniasis is an occupational hazard, the use of repellents such as such as DEET (N, N-diethyl3-toluamide), DEPA (N,N-diethylphenylacetamide) may be useful to prevent biting sandflies. Insecticide treated nets are useful in malaria control and are probably also useful for sandfly control in indoor transmission. An advantage of insecticide treated nets is that they can be integrated with malaria control as both diseases (VL and malaria) are found in the same general area in Ethiopia (FMoH, 2013). Indoor residual spraying is a simple and cost-effective method of controlling vector. Successful control has been practiced in country by complete destruction of the breeding/resting habitat of the sandfly (FMoH, 2013).

\section{Early diagnosis and management}

Early diagnosis and treatment are common methods to control VL in country for both individual patients and for the community (FMoH, 2013). Active case detection, surveillance and effective treatment, accompanied by measures for preventing re-infection, depending on the coverage achieved, should reduce or eliminate the parasite load and reduce transmission (WHO, 2010).

In Humera, about $30 \%$ of all VL patients are coinfected with HIV. Due to lower efficacy of antileishmanial drugs in HIV/VL extended treatment courses may be required to achieve cure (FMoH, 2013). Public education and mobilization

Multidisciplinary working group is organized as 'One Health Approach', in the country to eliminate VL. Partnership and collaboration are established with various stakeholders and other vector-borne disease control program. The target group for health education might include public health managers, health staff, community health workers and leaders, people living in endemic areas and patients (FMoH, 2013; Mengesha $e t$ al., 2014)). Health education is a core element in implementation of any disease prevention and control program. Communication is a key component of community empowerment and mobilization (FMoH, 2013). Social values change over time and vary in different communities and so may determine the acceptability of a given adaptation option. Disease mapping contributes to facilitate implementation of evidence-based integrated disease control activities at country and/or regional levels are also established (Seid et al., 2014).

\section{Conclusion}

Leishmaniasis is a serious neglected zoonotic disease with one or more reservoir hosts maintaining the disease. Among a limited range of reservoir hosts for leishmaniasis which can include dogs, hyraxes, rodents, wild canids and bats in Ethiopia. Currently, leishmaniasis has a wider geographical distribution pattern than before, and it is considered to be a growing public health concern, and this is mainly due to a number of risk factors such as environmental change, HIV co-infection, demographic and human behavior contribute to the changing landscape for zoonotic cutaneous and visceral leishmaniasis. The existing major agricultural schemes, ongoing and planned mega-projects such as sugar industries and sugar cane farming, dams for irrigation purposes and expansion of irrigation and/or rain fed agriculture all exist adjacent the endemic of VL within the lower Kola to Weina Dega agro-ecological zones. Those mega projects are suitable for reproduction of stray dogs, rodents, wild canids and vectors. Recent spread beyond the Metema and Humera plains to villages in Tahtay Adiabo, Sheraro and Welkait in the northwestern Tigray and became the most important focus in the epidemiology of VL. This new focus established in Tahtay Adiabo and Sheraro district might be due to the existing new agricultural irrigation for Welkait sugar factory. Moreover, there is limited awareness of the disease among local community and migrants to work in agricultural farms in endemic and epidemic regions. Therefore, special consideration such as carry out new research, control and prevention measures should be taken especially in and around the mega projects.

\section{Conflict of interest}

The authors declare that there is no conflict of interest.

References
Alemayehu, B. and Alemayehu, M. 2017.
Leishmaniasis: A Review on Parasite, Vector and Reservoir Host. Health Sci J. 11(4), 519.

Ali, A. and Ashford, R.W. 1994. Visceral leishmaniasis in Ethiopia I. Cross-sectional leishmanin skin test in an endemic locality. Ann. Trop. Med. Parasitol. 87, 157-161.

Al-Salem, W., Herricks, R.J. and Hotez, J.P. 2016. A review of visceral leishmaniasis during the conflict 
in South Sudan and the consequences for East African countries. Parasit. Vectors 9, 460.

Alvar, J., Velez, I.D., Bern, C., Herrero, M., Desjeux, P., Cano, J., Jannin, J. and den Boer, M. 2012. Leishmaniasis worldwide and global estimates of its incidence. PLoS One 7, e35671. doi: 10.1371/journal.pone.0035671.

Argaw, D., Mulugeta, A., Herrero, M., Nombela, N., Teklu, T., Tefera, T., Belew, Z., Alvar, J. and Bern, C. 2013. Risk factors for visceral leishmaniasis among residents and migrants in Kafta-Humera, Ethiopia. PLoS. Negl. Trop. Dis. 7, e2543. doi: 10.1371/journal.pntd.0002543.

Ashford, R., Bray, M., Hutchinson, M. and Bray, R. 1973. The Epidemiology of cutaneous leishmaniasis in Ethiopia. Trans. R. Soc. Trop. Med. Hyg. 67, 569-601.

Assimina, Z., Charilaos, K. and Fotoula, B. 2008. Leishmaniasis: an overlooked public health concern. Health Sci. J. 2, 196-205.

Berhe, M., Bsrat, A., Taddele, H., Gadissa, E., Hagos, Y., Tekle, Y. and Abera, A. 2018. Knowledge Attitude and Practice towards Visceral Leishmaniasis among Residents and Health Professionals in Welkait District, Western Tigray, Ethiopia. J. Trop. Dis. 6, 257. doi: 10.4172/2329891X.1000257.

Bsrat, A., Berhe, N., Balkew, M., Yohannes, M., Teklu, T., Gadisa, E., Medhin, G. and Abera, A. 2015. Epidemiological study of cutaneous leishmaniasis in Saesie Tsaeda-emba district, eastern Tigray, northern Ethiopia. Parasit. Vectors 8, 149. doi: 10.1186/s13071-015-0758-9.

CDC. 2013. Parasites - Leishmaniasis. Centers for Disease Control and Prevention. Available at: http://www.cdc.gov/parasites/leishmaniasis/. Accessed January 2017.

CFSPH. 2009. Leishmaniasis (cutaneous and visceral). Center for Food Security and Public Health, Iowa State of University, College of Veterinary Medicine, Iowa.

Chappuis, F., Sundar, S., Hailu, A., Ghalib, H., Rijal, S., Peeling, R.W., Alvar, J. and Boelaert, M. 2007. Visceral leishmaniasis: What are the needs for diagnosis, treatment and control? Nat. Rev. Microbiol. 5, 7-16.

Collin, S.M., Coleman, P.G., Ritmeijer, K. and Davidson, R.N .2006. Unseen Kala-azar deaths in south Sudan (1999-2002). Trop. Med. Int. Health $11,509-512$.

Coura-Vital, C.W., Reis, A.B., Reis, L.E., Braga, S.L., Roatt, B.M. and Aguiar-Soares, R.O. 2013. Canine visceral leishmaniasis: incidence and risk factors for infection in a cohort study in Brazil. Vet. Parasitol. 197, 411-417.
Dawit, G., Girma, Z. and Simenew, K. 2013. A Review on Biology, Epidemiology and Public Health Significance of Leishmaniasis. J. Bacteriol. Parasitol. 4, 166. doi: 10.4172/2155-9597.1000166.

Dereure, J., Boni, M., Pratlong, F., Osman, M., Boucheton, B. and Dedet, J.P. 2000. Visceral Leishmaniasis in Sudan: First identification of Leishmania from dogs. Trans. Roy. Soc. Trop. Med. Hyg. 94, 154-155.

Desjeux, P. 2004. Leishmaniasis: current situation and new perspectives. Comp. Immunol. Microb. 27, 305-318.

FMoH (Federal Ministry of Health). 2013. Guideline for diagnosis, treatment and prevention of leishmaniasis in Ethiopia. Edited by Unit NTD, $2^{\text {nd }}$ ed. Ethiopian Federal Ministry of Health; Addis Ababa, Ethiopia.

Fuller, G.K., Lemma, A., Haile, T. and Atwood, C.L. 1976. Kala-azar in Ethiopia I: Leishmanin skin test in Setit Humera, a kala-azar endemic area in northwestern Ethiopia. Ann. Trop. Med. Parasitol. 70, 147-163.

Gadisa, E., Tsegaw, T., Abera, A., Elnaiem, D., Boer, M., Aseffa, A. and Jorge, A. 2015. Ecoepidemiology of visceral leishmaniasis in Ethiopia. Parasit. Vectors 8, 381. doi: 10.1186/s13071-0150987-y.

Gebre-Michael, T., Balkew, M., Alamirew,T., Gudeta, N. and Reta, M. 2007. Preliminary entomological observations in a highland area of Amhara region, northern Ethiopia, with epidemic visceral leishmaniasis. Ann. Trop. Med. Parasitol. 101, 367370.

Gebre-Michael, T., Balkew, M., Berhe, N., Hailu, A. and Mekonnen, Y. 2010. Further studies on the phlebotomine sandflies of the kala-azar endemic lowlands of Humera-Metema (north-west Ethiopia) with observations on their natural blood meal sources. Parasit. Vectors 3, 6. doi: 10.1186/17563305-3-6.

Gebresilassie, A., Kirstein, O.D., Yared, S., Aklilu, E., Moncaz, A., Tekie, H., Balkew, M., Warburg, A., Hailu, A. and Gebre-Michael, T. 2015. Species composition of phlebotomine sand flies and bionomics of Phlebotomus orientalis (Diptera: Psychodidae) in an endemic focus of visceral leishmaniasis in Tahtay Adiyabo district, Northern Ethiopia. Parasit. Vector 8, 248 . doi: 10.1186/s13071-015-0849-7.

Getachew, T., Tadesse, A., Yoseph, M., Zenebe, A., Abere, B., Woldnecherkos, A., Fetih, M., Tesfaye, H., Girma, T. and Dejuma, Y. 2006. Internal medicine lecture notes for health officers, Ethiopian in collaboration with the Ethiopia public health training initiative. The Carter Center, the Ethiopia 
Ministry of Health, and the Ethiopia Ministry of Education, pp: 56-63.

Getahun, M. and Belete, A. 2007. Visceral Leishmaniasis and HIV co-infection in patients admitted to Gondar university hospital, northwest Ethiopia. Ethiop. J. Health Dev. 21, 53-60.

Hailu, A., Gramiccia, M. and Kager, P.A. 2009. Visceral leishmaniasis in Aba-Roba, south-western Ethiopia: prevalence and incidence of active and subclinical infections. Ann. Trop. Med. Parasitol. 103, 659- 670.

Hailu, T., Yimer, M., Mulu, W. and Abera, B. 2016. Challenges in visceral leishmaniasis control and elimination in the developing countries: A review. J. Vector Borne Dis. 53, 193-198.

Herrador, Z., Gherasim, A., Jimenez, B.C., Granados, M., San Martín, J.V. and Aparicio, P. 2015. Epidemiological Changes in Leishmaniasis in Spain According to Hospitalization-Based Records, 1997- 2011: Raising Awareness towards Leishmaniasis in Non-HIV Patients. PLoS Negl. Trop. Dis. 9(3), e0003594. doi: 10.1371/journal.pntd.0003594.

Hide, M., Bucheton, B., Kamhawi, S., Bras-Gonçalves, R., Sundar, S., Lemesre, J.L. and Bañuls, A.L. 2007. Understanding Human Leishmaniasis: The need for an integrated approach in encyclopedia of infectious diseases Book of microbiology. John Wiley and Sons Inc.

Hurissa, Z., Gebre-Silassie, S., Hailu, W., Tefera, T., Lallo, D.G. and Cuevas, L.E. 2010. Clinical characteristics and treatment outcome of patients with visceral leishmaniasis and HIV co-infection in northwest Ethiopia. Trop. Med. Int. Health 15, 848855.

Kassahun, A., Sadlova, J., Dvorak, V., Kostalova, T., Rohousova, I., Frynta, D., Aghova, T., YasurLandau, D., Lemma, W., Hailu, A., Baneth, G., Warburg, A., Volf, P. and Votypka, J. 2015a. Detection of $L$. donovani and $L$. tropica in Ethiopian wild Rodents. Acta Trop. 145, 39-44.

Kassahun, A., Sadlova, J., Kostalova, T., Benda, P., Warburg, A., Hailu, A., Baneth, G., Volf, P. and Votypka, J. 2015b. Natural infection of bats with Leishmania in Ethiopia. Acta Trop. 150, 166-170. https://doi.org/10.1016/j.actatropica.2015.07.024.

Kenubih, A., Dagnachew, S., Almaw, G., Abebe, T., Takele, Y., Hailu, A. and Lemma, W. 2015. Preliminary survey of domestic animal visceral leishmaniasis and risk factors in north west Ethiopia. Trop. Med. Int. Health 20(2), 205-210. doi: 10.1111/tmi.12418.

Kishore, K., Kumar, V., Kesari, S., Dinesh, D.S., Kumar, A.J., Das, P. and Bhattacharya, S.K. 2006. Vector control in leishmaniasis. Indian J. Med. Res. 123(3), 467-472.
Lemma, W. 2008. Review article: hyraxes and leishmaniasis in Ethiopia. Ethiop. J. Health Biomed. Sci. 1, 63-69.

Lemma, W., Erenso, G., Gadisa, E., Balkew, M., Gebre-Michael, T. and Hailu, A. 2009. A zoonotic focus of cutaneous leishmaniasis in Addis Ababa, Ethiopia. Parasit Vectors 2, 60. doi: 10.1186/17563305-2-60.

Lemma, W., Tekie, H., Balkew, M., Gebre-Michael, T., Warburg, A. and Hailu, A. 2014. Population dynamics and habitat preferences of Phlebotomus orientalis in extra-domestic habitats of Kafta Humera lowlands-kala azar endemic areas in Northwest Ethiopia. Parasit. Vectors 7, 359. doi: 10.1186/1756-3305-7-359.

Lemma, W., Tekie, H., Yared, S., Balkew, M., GebreMichael, T., Warburg, A. and Hailu, A. 2015. Seroprevalence of Leishmania donovani infection in labour migrants and entomological risk factors in extra-domestic habitats of Kafta-Humera lowlands - kala-azar endemic areas in the northwest Ethiopia. BMC Infect. Dis. 15, 99. doi: 10.1186/s12879-0150830-2.

Lemma, W. 2018. Zoonotic leishmaniases and controls in Ethiopia. Asian Pac. J. Trop. Med. 11(5), 313319.

Leta, S., Dao, T.H., Mesele, F. and Alemayehu, G. 2014. Visceral Leishmaniasis in Ethiopia: An Evolving Disease. PLoS Negl. Trop. Dis. 8(9), e3131. doi:10.1371/journal.pntd.0003131.

Malaria Consortium. 2010. Leishmaniasis control in eastern Africa: Past and present efforts and future needs. Situation and gap analysis.

Mengesha, B., Endris, M., Takele, Y., Mekonnen, K., Tadesse, T., Feleke, A. and Diro, E. 2014. Prevalence of malnutrition and associated risk factors among adult visceral leishmaniasis patients in Northwest Ethiopia: A cross sectional study. BMC Res. Notes 7(75), 75-77.

Moncaz, A., Gebresilassie, A., Kirstein, O., Faiman, R., Gebre-Michael, T., Hailu, A. and Warburg, A. 2013. Attraction of phlebotomine sand flies to baited and non-baited horizontal surfaces. Acta Trop. 126, 205-210.

Murray, H.W., Berman, J.D., Davies, C.R. and Saravia, N.G. 2005. Advances in leishmaniasis. Lancet 366, 1561-1577.

Negera, E., Gadisa, E., Yamuah, L., Engers, H., Hussein, J., Kuru, T., Hailu, A., Gedamu, L. and Aseffa, A. 2008. Outbreak of cutaneous leishmaniasis in Silti woreda, Ethiopia: risk factor assessment and causative agent identification. Trans. R. Soc. Trop. Med. Hyg. 102, 883-890.

Oryan, A., Alidadi, S. and Akbari, M. 2014. Risk Factors Associated With Leishmaniasis. Trop. Med. Surg. 2, 3 . 
Oryan, A. and Akbari, M. 2016. Worldwide risk factors in Leishmaniasis. A review. Asian Pac. J. Trop. Med. 9(10), 925-932.

Ready, P.D. 2008. Leishmaniasis emergence and climate change. Rev. Sci. Tech. Off. Int. Epi. 27(2), 399-412.

Reithinger, R., Mohsen, M. and Leslie, T. 2010. Risk factors for anthroponotic cutaneous Leishmaniasis at the household level in Kabul, Afghanistan. PLoS Negl. Trop. Dis. 4, e639. doi: 10.1371/journal.pntd.0000639.

Savoia, D. 2015. Recent updates and perspectives on leishmaniasis. J. Infect. Dev. Count. 9(6), 588-596.

Seid, A., Gadisa, E., Tsegaw, T., Abera, A., Teshome, A., Mulugeta, A., Herrero, M., Argaw, D., Jorge, A., Kebede, A. and Aseffa, A. 2014. Risk map for cutaneous leishmaniasis in Ethiopia based on environmental factors as revealed by geographical information systems and statistics. Geospat. Health 8, 377-387.

Shabtai, I., Shenker, M., Edeto, W., Warburg, A. and Ben-Hur, M. 2014. Effects of land use on structure and hydraulic properties of Vertisols containing a sodic horizon in northern Ethiopia. Soil Till. Res. 136, 19-27.

Shiferaw, Y., Wondimeneh, Y., Wondifraw, H. and Ferede, G. 2016. Trend Analysis of Visceral Leishmaniasis in Metema Hospital Northwest, Ethiopia. J. Epidemiol. Public Health Rev. 1(5), doi http://dx.doi.org/10.16966/2471-8211.129.

Spear, C.R. 2017. Review of "Mathematical Models for Neglected Tropical Diseases: Essential Tools for Control and Elimination. Parasit. Vectors 10, 38. doi: 10.1186/s13071-017-1974-2.

Tsegaw, T., Gadisa, E., Seid, A., Abera, A. and Teshome, A. 2013. Identification of environmental parameters and risk mapping of visceral leishmaniasis in Ethiopia by using geographical information systems and a statistical approach. Geospat. Health 7, 299-308.

WHO. 2010. Control of the leishmaniases. Report of a meeting of the WHO (World Health Organization) Expert Committee on the Control of Leishmaniases, 22-26 March 2010, Geneva, pp: 5-88.

WHO. 2014. Leishmaniasis. Fact sheet No. 375. World Health Organization, Geneva, 2014. Available at: http://www.who.int/mediacentre/factsheets/fs375/e n/ (Accessed on January 30, 2017).

WHO. 2015. Leishmaniasis. Fact sheet. World Health Organization, Geneva, 2015. Available at: http://www.who.int/mediacentre/factsheets/fs375/e $\mathrm{n} /$ (Accessed on January 20, 2018).

WHO. 2017. Leishmaniasis Ethiopia profile- 2015. World Health Organization, Geneva, 2017.

Wondimeneh, Y., Takele, Y., Atnafu, A., Ferede, G. and Muluye, D. 2014. Trend Analysis of Visceral Leishmaniasis at Addis Zemen Health Center, Northwest Ethiopia. Biomed. Res. Int. doi: $10.1155 / 2014 / 545393$.

Yared, S., Deribe, K., Gebreselassie, A., Lemma, W., Akililu, E., Kirstein, O.D., Balkew, M., Warburg, A., Gebre-Michael, T. and Hailu, A. 2014. Risk factors of Visceral leishmaniasis: a case control study in Northwest Ethiopia. Parasit. Vectors 7, 470. doi: 10.1186/s13071-014-0470-1.

Zackay, A., Nasereddin, A., Takele, Y., Tadesse, D., Hailu, W., Hurissa, Z., Yifru, S., Weldegebreal, T., Diro, E., Kassahun, A., Hailu, A. and Jaffe, C.L. 2013. Polymorphism in the HASPB repeat region of east African Leishmania donovani strains. PLoS Negl. Trop. Dis. 7, e2031. doi: 10.1371/journal.pntd.0002031. 